\title{
Risk assessment of exposure to volatile organic compounds in groundwater in Taiwan
}

\author{
Chihhao Fan ${ }^{a}$, Gen-Shuh Wang ${ }^{b}$, Yen-Chuan Chen ${ }^{c}$, Chun-Han Ko ${ }^{d, *}$ \\ aDepartment of Safety, Health, and Environmental Engineering, Mingchi University of Technology, Taipei County, Taiwan \\ bepartment of Public Health, National Taiwan University, Taipei, Taiwan \\ ${ }^{c}$ Energy and Environment Research Laboratories, Industrial Technology Research Institute, Hsinchu County, Taiwan \\ ${ }^{\mathrm{d}}$ School of Forest and Resources Conservation, National Taiwan University, Taipei, Taiwan
}

\section{A R T I C L E D A T A}

Article history:

Received 23 June 2008

Received in revised form

4 December 2008

Accepted 5 December 2008

Available online 22 January 2009

Keywords:

Risk assessment

Volatile organic compound

Groundwater

Cancer risk

\begin{abstract}
A B S T R A C T
The purpose of this study is to assess the risks from exposure to 14 volatile organic compounds (VOCs) in selected groundwater sites in Taiwan. The study employs the multimedia environment pollutant assessment system (MEPAS) model to calculate the specific non-cancer and cancer risks at an exposure level of $1 \mu \mathrm{g} / \mathrm{L}$ of each VOC for a variety of exposure pathways. The results show that the highest specific non-cancer risk is associated with water ingestion of vinyl chloride (VC) and that the highest specific cancer risk is associated with indoor breathing of VC. The three most important exposure pathways for risk assessment for both non-cancer and cancer risks are identified as water ingestion, dermal absorption when showering, and indoor breathing. Excess tetrachloroethylene (PCE), trichloroethylene (TCE), dichloroethylene (DCE), and VC are detected in the groundwater aquifers of one dump site and one factory. However, the study suggests that the pollutants in the contaminated groundwater aquifers do not travel extensively with groundwater flow and that the resulting VOC concentrations are below detectable levels for most of the sampled drinking-water treatment plants. Nevertheless, the non-cancer and cancer risks resulting from use of the contaminated groundwater are found to be hundred times higher than the general risk guidance values. To ensure safe groundwater utilisation, remediation initiatives for soil and groundwater are required. Finally, the study suggests that the current criteria for VOCs in drinking water might not be capable of ensuring public safety when groundwater is used as the primary water supply; more stringent quality criteria for drinking water are proposed for selected VOCs.
\end{abstract}

C 2009 Elsevier B.V. All rights reserved.

\section{Introduction}

Volatile organic compounds (VOCs) constitute a group of chemicals that vapourise easily and enter the atmosphere under normal conditions. Due to their volatility at low temperatures and their capacity for rapid dissolution in nonpolar solvents, VOCs have been used extensively for a range of industrial processes-including metal surface degreasing, textile cleansing, food extraction, and so on. However, improper storage, decanting, and application during manufacturing processes can cause serious environmental issues (Liang and Liao, 2007). VOCs have frequently been found in industrial and municipal wastes (Edil, 2003), and inadequate treatment and inappropriate disposal of industrial waste have been associated with leakages and emissions that are potentially harmful to human health and the natural environment (Malherbe and Mandin, 2007; Guo et al., 2004). In this regard, it is important to note that several VOCs are proven

\footnotetext{
* Corresponding author. Tel.: +88 6233664615; fax: +88 6223654520.

E-mail address: chunhank@ntu.edu.tw (C.-H. Ko).
} 
carcinogens that pose a demonstrable threat to public health (Belpomme et al., 2007; Claxton and Woodall, 2007).

Among the many environmental problems associated with VOCs, groundwater contamination has received much attention. VOCs have the potential to cause serious deterioration of soil and groundwater quality (Kistemann et al., 2007; Hohener et al., 2003), and this threat to public health is especially significant in areas where groundwater is the primary source for public water supplies. Although many remedial initiatives have been undertaken to reduce the various threats to public health posed by VOCs (Ellis and Rivett, 2007; Barry et al., 2002), groundwater remediation is complicated and time-consuming. Many times, using current groundwater criteria as the remediation goals leads to poor protection for the public. Moreover, even if most of the contaminants are successfully removed, residual contaminants can still pose a risk to the public and the environment. Therefore, risk management principles should be applied to establishing the site remediation goals.

Accurate risk assessment and ongoing risk management thus become important factors in civic programs to minimise potential threats to the public (Posen et al., 2006). Examples of relevant risk-assessment studies in the literature include: (i) estimation of the cancer risk from inhalation and dermal absorption of chloroform when showering in Taiwan (Kuo et al., 1998); (ii) estimation of the cancer risk from trihalomethanes in drinking water and inhalation exposure to chloroform (Wang et al., 2007); and (iii) estimation of the risks from exposure to trichlorethylene (TCE) and perchloroethylene (PCE) in contaminated groundwater in Taiwan (Chen and Ma, 2006). A review of these and other reported risk-assessment studies reveals that the accuracy of risk assessments varies-depending upon the models that are utilised and the exposure pathways that are taken into consideration. In assessing VOC risk, the selection of exposure pathways is of crucial importance because consideration of inappropriate exposure pathways can cause the risk to human health to be dangerously underestimated. In the context of risk assessment of VOCs in groundwater, all possible modes of VOC transmission must therefore be taken into consideration, and the models used for determining risk must include all relevant factors to ensure that the risk is accurately assessed.

All of this is of special significance in Taiwan, where groundwater is utilised as the major source of public water supplies in many rural and suburban areas and where a lack of proper attention to industrial storage and waste disposal has caused severe VOC contamination in groundwater (TEPA, 2005b). Although strenuous efforts have been made to implement groundwater remediation projects, and although the concentrations of contaminants have been gradually reduced, residual VOCs in groundwater still pose an appreciable threat to public health in Taiwan.

Against this background, the purpose of the present study is to assess health risks from exposure to several VOCs in the groundwater of Taiwan. Groundwater samples adjacent to several contamination remediation sites are collected and analysed for VOC concentrations. These are compared with samples from neighbouring drinking-water treatment plants that utilise groundwater as the primary water source. Various exposure pathways are investigated and the associated risks are calculated in accordance with the multimedia environment pollutant assessment system (MEPAS). The study concludes with a set of proposed criteria for groundwater VOCs.

\section{Materials and methods}

\subsection{Sample collection and analysis}

As shown in Fig. 1, groundwater samples were collected from wells adjacent to eight groundwater contamination remediation sites in Taiwan (designated 'S1-S8' in Fig. 1). The eight remediation sites included four illegal dump sites (S1, S2, S3, and S5), one gas station (S4), one disused factory (S6), one trichloroethylene production factory (S7), and one licensed dump site (S8). The distances between the wells and the groundwater contamination remediation sites ranged from $0 \mathrm{~m}$ to $50 \mathrm{~m}$, with an average of $5 \mathrm{~m}$.

For comparison with the samples from the contamination remediation sites, groundwater samples were also collected from ten neighbouring drinking-water treatment plants (designated 'W1-W10' in Fig. 1). These treatment plants, which were down-gradient from the contamination remediation sites, used groundwater as source water. The samples were taken prior to water treatment. The average distance between the drinking-water treatment plants and the groundwater contamination remediation sites was $12 \mathrm{~km}$.

The subsurface hydraulic conductivities in the investigated areas ranged from $10^{-6}$ to $10^{-4} \mathrm{~m} / \mathrm{s}$ (MOEA, 1997). The groundwater samples from each location were collected for VOC analysis on three separate occasions (April 2005, July 2005, and October 2005).

To assess the potential health risk from exposure to VOCs in the groundwater, the samples were tested for fourteen

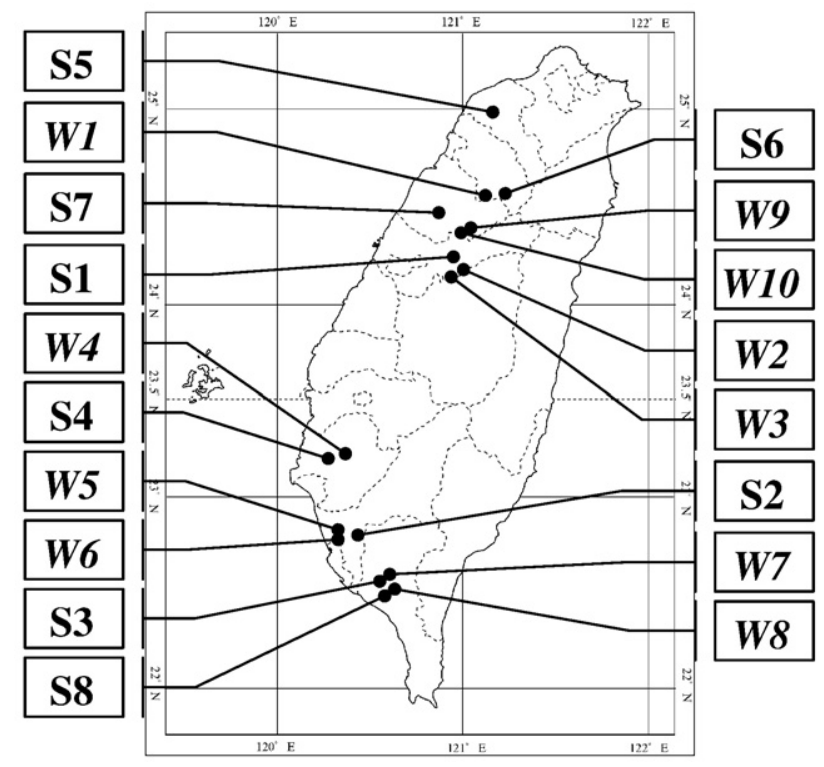

Fig. 1-Geographical distribution of sampling locations in the study (S: contamination sites; W: drinking-water treatment plants). 
possible VOCs: (i) tetrachloroethylene (otherwise known as perchloroethylene, PCE); (ii) trichloroethylene (TCE); (iii) 1,1dichloroethylene (1,1-DCE); (iv) cis-1,2-dichloroethylene (cis1,2-DCE); (v) trans-1,2-dichloroethylene (trans-1,2-DCE); (vi) vinyl chloride (VC); (vii) 1,1-dichloroethane (1,1-DCEA); (viii) benzene (BENZ); (ix) toluene (TLN) (all three isomers); $(\mathrm{x})$ xylene (XYL); (xi) 1,2-dichlorobenzene (1,2-DCB); (xi) ethylbenzene (EBENZ); (xii) styrene (STY); and (xiii) methyl-t-butyl ether (MTBE). Of these, TCE, 1,1-DCE, VC, and BENZ are included in the Taiwan quality criteria for drinking water as potential threats to human health. The remaining 11 VOCs were selected for analysis because their presence in groundwater has been reported to have significant impacts on public health and the environment (TEPA, 2005a,b). Selected physical and chemical properties of the 14 VOCs investigated in the study are presented in Table 1.

All the sampling processes followed the protocols of the Taiwan Environmental Protection Administration (TEPA) for sampling drinking water for VOC analysis (EALTEPA, 2008). In each instance, duplicate water samples were collected and placed in pre-cleaned $40-\mathrm{mL}$ amber glass bottles with Teflonlined rubber septa; these were then sealed and stored at $4{ }^{\circ} \mathrm{C}$ before analysis. All VOC analyses were performed within seven days of sampling.

The determination of VOC concentration was conducted in accordance with the standard method promulgated by TEPA (NIEA W785.54B). The analytical equipment consisted of a purge-and-trap system (4552 Water/Soil Autosampler and 4660 Sample Concentrator by IO Analytical) connected to GC/ MS (GC 6890N/5973N MSD by Agilent) with a fused silica SPB-624 capillary column (RTX-Volatiles, $60 \mathrm{~m} \times 0.32 \mathrm{~mm} \times 1.8 \mu \mathrm{m}$ ). Quality control (QC) samples were prepared and analysed for each batch of samples. The QC results showed that the deviation between duplicates was within $20 \%$ and that the recovery of laboratory control standards was between $90 \%$ and $110 \%$.

\subsection{Risk-assessment model}

The multimedia environment pollutant assessment system (MEPAS), which was originally developed by the Pacific
Northwest National Laboratory of the US Department of Energy in 1995, was employed to perform the risk assessment because it has the most complete set of exposure pathways of all popular multimedia models. MEPAS assesses the health risks resulting from environmental pathways and human exposure through higher-order mathematical calculations. Although MEPAS is most commonly used for risk assessment of soil and groundwater contamination sites, it can also be used for risk assessment of contamination sources in bodies of surface water and in the atmosphere.

MEPAS consists of three calculation modules:

${ }^{*}$ module of contamination sources;

* module of contact pathways (including overland pathways, vadose and saturated underground aquifers, surface water, atmosphere, inhalation, ingestion, and dermal exposure); and

* module of cancer, non-cancer, and radioactive risk calculation.

Using MEPAS, human risk is calculated by following equation (USEPA, 1989):

$$
\begin{aligned}
\text { CancerRisk } & =\sum_{k}\left[1-\exp \left(\mathrm{ADI}_{k} \times \mathrm{CSF}_{k}\right)\right] \\
& \approx \sum_{k} \mathrm{ADI}_{k} \times \mathrm{CSF}_{k}
\end{aligned}
$$

in which:

$\mathrm{ADI}_{k}$ the average daily intake $\left(\mathrm{mg} \mathrm{kg}^{-1} \mathrm{day}^{-1}\right)$ of contaminants by receptors through exposure route $\mathrm{k}$ (that is, ingestion, inhalation, or dermal absorption); and

$\mathrm{CSF}_{k}$ the cancer slope factor (kg day $\mathrm{mg}^{-1}$ ) of exposure route $k$, which is derived from dose-response studies.

In practice, the approximation in the above equation is used for small values of average daily intake and cancer slope factor $(\mathrm{ADI} \times \mathrm{CSF})$, in which range the risk is linearly proportional to the contaminant concentration exposed.

\begin{tabular}{|c|c|c|c|c|}
\hline VOC & Chemical formula & Abbreviation & Molecular weight & $\log K_{\text {ow }}^{\mathrm{a}}$ \\
\hline Tetrachloroethylene & $\mathrm{CCl}_{2} \mathrm{CCl}_{2}$ & PCE & 166 & 3.40 \\
\hline Trichloroethylene & $\mathrm{CHClCCl}_{2}$ & TCE & 131.5 & 2.29 \\
\hline 1,1-dichloroethylene & $\mathrm{CH}_{2} \mathrm{CCl}_{2}$ & 1,1-DCE & 97 & 1.32 \\
\hline cis-1,2-dichloroethylene & $\mathrm{CHClCHCl}$ & cis-1,2-DCE & 97 & 1.86 \\
\hline trans-1,2-dichloroethylene & $\mathrm{CHClCHCl}$ & trans-1,2-DCE & 97 & 2.06 \\
\hline Vinyl chloride & $\mathrm{CHClCH}_{2}$ & VC & 62.5 & 0.60 \\
\hline 1,1-dichloroethane & $\mathrm{CH}_{3} \mathrm{CHCl}_{2}$ & 1,1-DCEA & 99 & - \\
\hline Benzene & $\mathrm{C}_{6} \mathrm{H}_{6}$ & BENZ & 78 & 2.13 \\
\hline Toluene & $\mathrm{CH}_{3} \mathrm{C}_{6} \mathrm{H}_{5}$ & TLN & 92 & 2.69 \\
\hline Xylene & $\mathrm{CH}_{3} \mathrm{C}_{6} \mathrm{H}_{4} \mathrm{CH}_{3}$ & XYL & 106 & 3.18 \\
\hline 1,2-dichlorobenzene & $\mathrm{Cl}_{2} \mathrm{C}_{6} \mathrm{H}_{4}$ & 1,2-DCB & 147 & 3.38 \\
\hline Ethylbenzene & $\mathrm{CH}_{3} \mathrm{CH}_{2} \mathrm{C}_{6} \mathrm{H}_{5}$ & EBENZ & 106 & 3.15 \\
\hline Styrene & $\mathrm{C}_{2} \mathrm{H}_{3} \mathrm{C}_{6} \mathrm{H}_{5}$ & STY & 104 & 2.95 \\
\hline Methyl-t-butyl Ether & $\mathrm{C}_{5} \mathrm{H}_{12} \mathrm{O}$ & MTBE & 88 & 1.06 \\
\hline
\end{tabular}

Table 1 - Chemical formulae, abbreviations, molecular weights, and $K_{\text {ow }}$ values of the 14 Vocs analysed in the study 
ADI is the amount of contaminants received by human bodies via all exposure pathways. It is evaluated by the following equation (Mckone, 1993):

$\mathrm{ADI}_{i j k}=C_{i} \times \frac{C_{j}}{C_{i}} \times \frac{\mathrm{CR}_{i j k}}{\mathrm{BW}} \times \frac{\mathrm{EF} \times \mathrm{ED}}{\mathrm{AT}}$

in which:

$\mathrm{ADI}_{i j k}\left(\mathrm{mg} \mathrm{kg}^{-1}\right.$ day $\left.^{-1}\right)$ the average daily intake from environmental media i (air, soil, or groundwater), exposure media $j$ (drinking water, food, etc.), and exposure route $k$ (inhalation, ingestion, or dermal absorption);

$C_{i} \quad$ the pollutant concentration in environmental media $i$;

$C_{j} \quad$ the pollutant concentration in exposure media $j$;

$\mathrm{CR}_{i j k}$ the contact rate of exposure media $j$ through exposure route $k$ in environmental media $i$;

EF the exposure frequency;

ED the exposure duration;

AT the average time; and

BW the body weight of the receptor.

\subsection{Risk calculation}

The specific risk posed by a given aqueous chemical through a selected exposure pathway was calculated on the assumption of exposure to $1 \mu \mathrm{g} / \mathrm{L}$ (aqueous concentration) of that chemical. The exposure pathways that were investigated were categorised into three groups: (i) oral ingestion (ingestion of drinking water, showering water, meat, vegetables, crops, and soils); (ii) dermal absorption (showering and soil contact); and (iii) inhalation (indoor breathing and breathing when showering). The parameters used in the exposure assessment are presented in Table 2 .

Having calculated the specific risks and collected the VOC data, a risk assessment was conducted to evaluate the risk (both cancer risk and non-cancer risk) resulting from potential VOC uptake by means of oral ingestion, inhalation, and dermal absorption, as follows:

$$
\begin{aligned}
& \text { Risk }_{\mathrm{VOC} i}=\text { Risk }_{\mathrm{VOC}} \text {,ingestion }+ \text { Risk }_{\mathrm{VOC} i, \text { inhalation }}+\text { Risk }_{\mathrm{VOC} i, \text { dermal }} \\
& \text { Risk }_{\mathrm{VOC}} \text {, group A }=\Sigma\left(\text { Risk }_{\mathrm{VOC}} \text { i,pathway of group } \mathrm{A}\right) \\
& =\Sigma\left(\left(\text { Specific Risk } \mathrm{VOC}_{\mathrm{i}} \text {, pathway of group } \mathrm{A}\right) \times \mathrm{C}_{\mathrm{i}}\right)
\end{aligned}
$$

in which:

$C_{i} \quad$ aqueous concentration of VOC $i$.

\section{Results and discussion}

\subsection{Concentrations of VOCs in groundwater}

A total of 54 groundwater samples were analysed. No VOCs were detected in 45 of these. In the remaining 9 samples, which were collected from five sites (S2, S6, S7, S8, and W2), only PCE, TCE, 1,1-DCE, cis-1,2-DCE, VC, 1,1-DCEA, BENZ, TLN, and 1,2-DCB were detected. Table 3 shows the dates of sampling and concentrations of the detected VOCs from

\begin{tabular}{|c|c|c|}
\hline Parameters & Definitions & Values \\
\hline \multicolumn{3}{|c|}{ Site characteristic parameters } \\
\hline IR & Irrigation frequency $\left(\mathrm{L} / \mathrm{m}^{2} / \mathrm{mon}\right)$ & $0.841^{\mathrm{a}}$ \\
\hline$\rho_{\mathrm{b}}$ & Surface soil density $\left(\mathrm{g} / \mathrm{cm}^{3}\right)$ & $1.5^{\mathrm{a}}$ \\
\hline Tdd & Soil depth of contamination (m) & $0.1^{\mathrm{a}}$ \\
\hline$P$ & Soil surface density $\left(\mathrm{kg} / \mathrm{m}^{2}\right)$ & $240.0^{b}$ \\
\hline \multicolumn{3}{|c|}{ Food chain parameters } \\
\hline TVlv & $\begin{array}{l}\text { Plant surface conductive factor } \\
\text { (unitless) }\end{array}$ & $0.9^{\mathrm{b}}$ \\
\hline Rlv & $\begin{array}{l}\text { Plant surface sedimentation rate } \\
\text { (unitless) }\end{array}$ & $0.25^{\mathrm{b}}$ \\
\hline TClv & Vegetable growth period (day) & $60^{\mathrm{b}}$ \\
\hline Ylv & Specific vegetable yield $\left(\mathrm{kg} / \mathrm{m}^{2}\right)$ & $200^{\mathrm{b}}$ \\
\hline FIlv & $\begin{array}{l}\text { Yearly irrigation to vegetable ratio } \\
\text { (unitless) }\end{array}$ & $0.68^{b}$ \\
\hline Qft & Animal feed (kg/day) & $55.0^{\mathrm{C}}$ \\
\hline Qst & Animal soil intake (kg/day) & $0.5^{c}$ \\
\hline Qwt & Animal water intake (L/day) & $50^{c}$ \\
\hline \multicolumn{3}{|c|}{ Exposure parameters } \\
\hline $\mathrm{EF}$ & Exposure frequency (unitless) & $0.959^{d}$ \\
\hline ED & Exposure duration (yrs/lifetime) & $24^{\mathrm{d}}$ \\
\hline BW & Body weight $(\mathrm{kg})$ & $61.67^{d}$ \\
\hline AT & Average time (yrs) & $75.0(24.0)^{\mathrm{e}, \mathrm{d}}$ \\
\hline Asd & Body surface area $\left(\mathrm{cm}^{2}\right)$ & $1.73 \mathrm{E}+4^{\mathrm{d}}$ \\
\hline Add & Soil contact body surface area $\left(\mathrm{cm}^{2}\right)$ & $4.98 \mathrm{E}+3^{\mathrm{a}}$ \\
\hline $\mathrm{AD}$ & Soil viscous factor $\left(\mathrm{mg} / \mathrm{cm}^{2}\right)$ & $1.0^{\mathrm{b}}$ \\
\hline Kc & Indoor volatile factor $\left(\mathrm{L} / \mathrm{m}^{3}\right)$ & $0.5^{b}$ \\
\hline ETs & Daily shower duration (hrs/day) & $0.5^{\mathrm{d}}$ \\
\hline Udw & Daily water intake (L/day) & $3.0^{\mathrm{d}}$ \\
\hline Usw & $\begin{array}{l}\text { Daily water intake during showering } \\
\text { (L/day) }\end{array}$ & $0.06^{\mathrm{b}}$ \\
\hline Ulv & Daily vegetable intake (kg/day) & $0.323^{f}$ \\
\hline Ulr & Daily crop intake (kg/day) & $0.210^{f}$ \\
\hline Umt & Daily meat intake (kg/day) & $7.86 \mathrm{E}-3^{\mathrm{f}}$ \\
\hline Umk & Daily milk intake (L/day) & $5.59 \mathrm{E}-2^{\mathrm{f}}$ \\
\hline Usi & $\begin{array}{l}\text { Daily inhalation during showering } \\
\left(\mathrm{m}^{3} / \text { day }\right)\end{array}$ & $0.7^{\mathrm{d}}$ \\
\hline Uai & Daily inhalation indoors ( $\mathrm{m}^{3} /$ day) & $15.0^{\mathrm{b}}$ \\
\hline Uds & Daily soil intake (g/day) & $0.1^{\mathrm{d}}$ \\
\hline
\end{tabular}

Table 2 - Input parameters for site characteristics, food chain, and exposure assessment

a Ton (1999);

b Droppo et al. (1989);

c USEPA (1996);

d TEPA (2005c);

e the number in parenthesis denotes the value for non-cancer risk calculation.

f $\mathrm{DOH}(2000)$

these sites. In addition, Table 3 provides the detection limits and current drinking-water criteria for each detected VOC.

Individual VOCs were detected in 33 measurements. Of these, 15 revealed VOC concentrations above the current drinking-water criteria set by TEPA. With one exception, all of these involved chlorinated VOCs in groundwater from S7 (a TCE production factory) and S8 (a licensed dump site); the exception was one measurement of benzene from $\mathrm{S7}$.

Apart from these samples from S7 and S8, none of the many groundwater samples from contamination sites revealed any significant contamination (that is, concentrations that exceeded the water-quality criteria). In this regard, it is noteworthy that 
Table 3 - VOC concentrations in groundwater of the studied area

\begin{tabular}{|c|c|c|c|c|c|c|c|c|c|c|c|}
\hline \multirow[t]{2}{*}{ Category } & \multirow[t]{2}{*}{ Location } & \multirow{2}{*}{$\begin{array}{l}\text { Sampling } \\
\text { time }\end{array}$} & \multicolumn{9}{|c|}{$\operatorname{VOCs}(\mu \mathrm{g} / \mathrm{L})$} \\
\hline & & & PCE & TCE & 1,1-DCE & cis-1,2-DCE & VC & 1,1-DCEA & BENZ & TLN & 1,2-DCB \\
\hline \multirow[t]{7}{*}{ Contamination site } & S6 & April & - & 0.98 & - & - & - & - & - & - & - \\
\hline & S2 & April & - & - & - & - & - & - & - & 1 & - \\
\hline & S8 & April & 2 & 2 & 15 & 1 & - & 1 & - & - & - \\
\hline & & Jul. & 4 & 3 & 24 & 2 & - & 2 & - & - & - \\
\hline & S7 & April & 21 & 231 & 19 & - & 651 & 26 & 3 & - & 7 \\
\hline & & Jul. & 3.14 & 12.5 & 21.4 & - & 4.25 & 7.69 & - & - & - \\
\hline & & Oct. & 4.12 & 30.8 & 13 & 82.4 & 476 & 56.2 & 21.1 & - & - \\
\hline \multirow[t]{2}{*}{ Drinking-water treatment plant } & W2 & April & - & - & - & - & - & - & - & 1.63 & - \\
\hline & & Oct. & - & - & - & - & - & - & - & 20.2 & - \\
\hline Detection limit $(\mu \mathrm{g} / \mathrm{L})$ & & & 0.62 & 0.89 & 0.76 & 0.53 & 0.97 & 0.64 & 0.62 & 0.96 & 0.61 \\
\hline $\operatorname{Standard}(\mu \mathrm{g} / \mathrm{L})$ & & & $5^{a}$ & $5^{a}$ & $7^{\mathrm{a}}$ & $70^{\mathrm{a}}$ & $2^{a}$ & $50^{\mathrm{b}}$ & $5^{a}$ & $1^{\mathrm{c}}$ & $0.6^{\mathrm{c}}$ \\
\hline $\begin{array}{l}\text { "-” indicates the concentration lo } \\
\text { a Taiwan drinking water criteria } \\
\text { b New Jersey MCL. } \\
\text { c US } 2003 \text { MCL. }\end{array}$ & wer than $d$ & etection limit. & & & & & & & & & \\
\hline
\end{tabular}

the hydraulic conductivities of the surrounding aquifers ranged from $10^{-6}$ to $10^{-4} \mathrm{~m} / \mathrm{s}$; given these values, it is possible that any pollutants in the underground aquifer might have travelled very slowly or might have been confined within the contamination sites by remediation initiatives implemented in these locations.

The highest TCE and VC concentrations were found in the sample collected from S7 in April 2005. In this sample, the TCE concentration was 46 times the prescribed criterion for drinking water and the VC concentration was 326 times the drinking-water criterion. The abundance of TCE and VC in groundwater at this site has the potential to cause adverse public health effects if this water is employed for domestic use, and it is apparent that additional soil and groundwater remediation initiatives are urgently required to ensure the safety of groundwater utilisation in this location.

In the samples taken from drinking-water treatment plants (W1-W10), the only abnormality was the detection of a trivial amount of TLN in a sample from W2. It is thus apparent that the groundwater from the drinking-water treatment plants that were adjacent to contamination sites (that is, W9-10 adjacent to S7, and W7-8 adjacent to S8) had not been contaminated by pollutants from these contamination sites (or from any other VOC sources).

\subsection{Comparison of risks from different exposure pathways}

To assess the potential threats from daily utilisation of VOCcontaminated groundwater through various exposure pathways, the specific risks were calculated for each VOC for each pathway. The non-cancer risk was calculated for all VOCs; however, because only six of the fourteen investigated VOCs are considered carcinogenic (PCE, TCE, 1,1-DCE, VC, 1,1-DCEA, and BENZ), these were the only chemicals included in the cancer risk evaluation.

The specific risk of a given chemical was defined as the risk caused by exposure to $1 \mu \mathrm{g} / \mathrm{L}$ of that chemical through a particular pathway. In addition, the total risk for each chemical was calculated as the total of calculated risks from all exposure pathways. The results are presented in Tables 4 and 5 . The numbers in parentheses represent the relative risk compared with the risk from oral ingestion of PCE (taking the specific risk of oral water intake of PCE as unity).

\subsubsection{Specific non-cancer risk comparisons}

As shown in Table 4, VC, 1,1-DCE, and PCE were associated with the highest non-cancer risks, whereas XYL and MTBE posed the lowest non-cancer risks.

For VC, which represented the greatest non-cancer threat of all VOCs, the total estimated specific non-cancer risk was $8.39 \times 10^{-1}$. The most important exposure pathway for this chemical was water ingestion $\left(7.46 \times 10^{-1}\right)$. In contrast, noncancer risks from VC exposure through other pathways were either comparatively small (indoor breathing and dermal absorption when showering) or negligible (all other pathways). For 1,1-DCE, which represented the second-greatest noncancer risk of all VOCs, indoor inhalation and water ingestion were the two major pathways associated with non-cancer risk.

Comparisons of the various exposure pathways revealed that water ingestion, indoor breathing, and dermal absorption during showering were the three most important pathways for non-cancer risk. Of the various exposure pathways that involved oral ingestion, water intake resulted in the highest non-cancer risk, with consumption of vegetables and crops ranking second and third respectively; however, in general terms, it was apparent from the findings that VOC non-cancer risk via the food chain was negligible (apart from exposure by direct water consumption). Dermal absorption through soil contact and ingestion of meat, milk, and soil were found to have negligible influences on specific non-cancer risk for the VOCs studied here.

\subsubsection{Specific cancer risk comparisons}

As shown in Table 5, the relative cancer risk for 1,1-DCE, which was 20 times that of PCE, was the highest among the six carcinogenic VOCs that were assessed. VC (13 times that of PCE) and BENZ (six times that of PCE) had the second- and third-highest relative cancer risks. Only TCE and 1,1-DCEA had specific cancer risks that did not exceed the general guidance value $\left(10^{-6}\right)$. 
Table 4 - Specific non-cancer risk ${ }^{*}$ for VOCs through different pathways

\begin{tabular}{|c|c|c|c|c|c|c|c|c|c|c|c|c|c|c|}
\hline Pathway & PCE & TCE & 1,1-DCE & cis-1,2-DCE & trans-1,2-DCE & VC & 1,1-DCEA & BENZ & TLN & XYL & 1,2-DCB & EBENZ & STY & MTBE \\
\hline \multicolumn{15}{|l|}{ Ingestion } \\
\hline Water & $\begin{array}{l}1.49 \mathrm{E}-03 \\
(1.000)\end{array}$ & $\begin{array}{l}2.13 \mathrm{E}-03 \\
(1.430)\end{array}$ & $\begin{array}{l}1.66 \mathrm{E}-03 \\
(1.114)\end{array}$ & $\begin{array}{l}1.49 \mathrm{E}-03 \\
(1.000)\end{array}$ & $\begin{array}{l}7.46 \mathrm{E}-04 \\
(0.501)\end{array}$ & $\begin{array}{l}7.46 \mathrm{E}-01 \\
(500.671)\end{array}$ & $\begin{array}{l}1.49 \mathrm{E}-04 \\
(0.100)\end{array}$ & $\begin{array}{l}8.73 E-04 \\
(0.586)\end{array}$ & $\begin{array}{l}7.46 \mathrm{E}-05 \\
(0.050)\end{array}$ & $\begin{array}{l}7.46 \mathrm{E}-06 \\
(0.005)\end{array}$ & $\begin{array}{l}1.66 \mathrm{E}-04 \\
(0.111)\end{array}$ & $\begin{array}{l}1.49 \mathrm{E}-04 \\
(0.100)\end{array}$ & $\begin{array}{l}7.46 \mathrm{E}-05 \\
(0.050)\end{array}$ & $\begin{array}{l}4.98 \mathrm{E}-05 \\
(0.033)\end{array}$ \\
\hline Shower & $\begin{array}{l}1.49 \mathrm{E}-05 \\
(0.010)\end{array}$ & $\begin{array}{l}2.13 E-05 \\
(0.014)\end{array}$ & $\begin{array}{l}1.66 \mathrm{E}-05 \\
(0.011)\end{array}$ & $\begin{array}{l}1.49 \mathrm{E}-05 \\
(0.010)\end{array}$ & $\begin{array}{l}7.46 E-06 \\
(0.005)\end{array}$ & $\begin{array}{l}7.46 \mathrm{E}-03 \\
(5.007)\end{array}$ & $\begin{array}{l}1.49 \mathrm{E}-07 \\
(0.001)\end{array}$ & $\begin{array}{l}8.73 E-07 \\
(0.006)\end{array}$ & $\begin{array}{l}7.46 \mathrm{E}-07 \\
(0.001)\end{array}$ & $\begin{array}{l}7.46 \mathrm{E}-08 \\
(0.000)\end{array}$ & $\begin{array}{l}1.66 \mathrm{E}-07 \\
(0.001)\end{array}$ & $\begin{array}{l}1.49 \mathrm{E}-06 \\
(0.001)\end{array}$ & $\begin{array}{l}7.46 \mathrm{E}-07 \\
(0.001)\end{array}$ & $\begin{array}{l}4.98 \mathrm{E}-07 \\
(0.000)\end{array}$ \\
\hline Meat & $\begin{array}{l}\text { 6.04E-09 } \\
(0.000)\end{array}$ & $\begin{array}{l}8.40 \mathrm{E}-09 \\
(0.000)\end{array}$ & $\begin{array}{l}8.19 \mathrm{E}-10 \\
(0.000)\end{array}$ & $\begin{array}{l}2.84 \mathrm{E}-10 \\
(0.000)\end{array}$ & $\begin{array}{l}3.03 E-10 \\
(0.000)\end{array}$ & $\begin{array}{l}3.66 \mathrm{E}-07 \\
(0.000)\end{array}$ & $\begin{array}{l}3.15 E-11 \\
(0.000)\end{array}$ & $\begin{array}{l}4.65 \mathrm{E}-10 \\
(0.000)\end{array}$ & $\begin{array}{l}1.19 \mathrm{E}-10 \\
(0.000)\end{array}$ & $\begin{array}{l}3.20 \mathrm{E}-11 \\
(0.000)\end{array}$ & $\begin{array}{l}\text { 1.57E-09 } \\
(0.000)\end{array}$ & $\begin{array}{l}6.55 \mathrm{E}-10 \\
(0.000)\end{array}$ & $\begin{array}{l}2.20 \mathrm{E}-10 \\
(0.000)\end{array}$ & $\begin{array}{l}1.43 \mathrm{E}-12 \\
(0.000)\end{array}$ \\
\hline Milk & $\begin{array}{l}4.88 \mathrm{E}-09 \\
(0.000)\end{array}$ & $\begin{array}{l}\text { 6.59E-09 } \\
(0.000)\end{array}$ & $\begin{array}{l}1.84 \mathrm{E}-09 \\
(0.000)\end{array}$ & $\begin{array}{l}6.38 \mathrm{E}-10 \\
(0.000)\end{array}$ & $\begin{array}{l}6.82 E-10 \\
(0.000)\end{array}$ & $\begin{array}{l}8.23 \mathrm{E}-07 \\
(0.001)\end{array}$ & $\begin{array}{l}7.08 E-11 \\
(0.000)\end{array}$ & $\begin{array}{l}1.05 E-09 \\
(0.000)\end{array}$ & $\begin{array}{l}2.68 \mathrm{E}-10 \\
(0.000)\end{array}$ & $\begin{array}{l}7.19 \mathrm{E}-11 \\
(0.000)\end{array}$ & $\begin{array}{l}3.54 \mathrm{E}-09 \\
(0.000)\end{array}$ & $\begin{array}{l}1.47 \mathrm{E}-09 \\
(0.000)\end{array}$ & $\begin{array}{l}4.96 \mathrm{E}-10 \\
(0.000)\end{array}$ & $\begin{array}{l}3.22 \mathrm{E}-12 \\
(0.000)\end{array}$ \\
\hline Vegetables & $\begin{array}{l}2.85 \mathrm{E}-05 \\
(0.019)\end{array}$ & $\begin{array}{l}5.71 \mathrm{E}-05 \\
(0.038)\end{array}$ & $\begin{array}{l}2.95 \mathrm{E}-05 \\
(0.020)\end{array}$ & $\begin{array}{l}2.62 \mathrm{E}-05 \\
(0.018)\end{array}$ & $\begin{array}{l}8.86 E-06 \\
(0.006)\end{array}$ & $\begin{array}{l}1.27 \mathrm{E}-02 \\
(8.523)\end{array}$ & $\begin{array}{l}1.35 \mathrm{E}-06 \\
(0.001)\end{array}$ & $\begin{array}{l}1.21 \mathrm{E}-05 \\
(0.008)\end{array}$ & $\begin{array}{l}4.15 \mathrm{E}-07 \\
(0.000)\end{array}$ & $\begin{array}{l}3.05 \mathrm{E}-08 \\
(0.000)\end{array}$ & $\begin{array}{l}1.38 \mathrm{E}-06 \\
(0.001)\end{array}$ & $\begin{array}{l}3.70 \mathrm{E}-07 \\
(0.000)\end{array}$ & $\begin{array}{l}4.68 \mathrm{E}-07 \\
(0.000)\end{array}$ & $\begin{array}{l}2.54 \mathrm{E}-07 \\
(0.000)\end{array}$ \\
\hline Crops & $\begin{array}{l}2.53 \mathrm{E}-05 \\
(0.017)\end{array}$ & $\begin{array}{l}1.41 \mathrm{E}-04 \\
(0.095)\end{array}$ & $\begin{array}{l}1.67 \mathrm{E}-04 \\
(0.112)\end{array}$ & $\begin{array}{l}9.72 \mathrm{E}-06 \\
(0.007)\end{array}$ & $\begin{array}{l}2.31 \mathrm{E}-06 \\
(0.002)\end{array}$ & $\begin{array}{l}1.12 \mathrm{E}-02 \\
(7.517)\end{array}$ & $\begin{array}{l}1.44 \mathrm{E}-07 \\
(0.000)\end{array}$ & $\begin{array}{l}4.78 \mathrm{E}-06 \\
(0.003)\end{array}$ & $\begin{array}{l}4.71 \mathrm{E}-08 \\
(0.000)\end{array}$ & $\begin{array}{l}2.47 \mathrm{E}-09 \\
(0.000)\end{array}$ & $\begin{array}{l}9.57 \mathrm{E}-07 \\
(0.001)\end{array}$ & $\begin{array}{l}2.57 \mathrm{E}-08 \\
(0.000)\end{array}$ & $\begin{array}{l}1.68 \mathrm{E}-07 \\
(0.000)\end{array}$ & $\begin{array}{l}7.44 \mathrm{E}-08 \\
(0.000)\end{array}$ \\
\hline Soil & $\begin{array}{l}\text { 7.13E-09 } \\
(0.000)\end{array}$ & $\begin{array}{l}1.51 \mathrm{E}-08 \\
(0.000)\end{array}$ & $\begin{array}{l}3.95 E-10 \\
(0.000)\end{array}$ & $\begin{array}{l}2.04 \mathrm{E}-09 \\
(0.000)\end{array}$ & $\begin{array}{l}8.01 \mathrm{E}-10 \\
(0.000)\end{array}$ & $\begin{array}{l}1.78 \mathrm{E}-06 \\
(0.001)\end{array}$ & $\begin{array}{l}6.72 \mathrm{E}-11 \\
(0.000)\end{array}$ & $\begin{array}{l}1.45 \mathrm{E}-09 \\
(0.000)\end{array}$ & $\begin{array}{l}1.89 \mathrm{E}--11 \\
(0.000)\end{array}$ & $\begin{array}{l}1.01 \mathrm{E}-12 \\
(0.000)\end{array}$ & $\begin{array}{l}3.18 \mathrm{E}-10 \\
(0.000)\end{array}$ & $\begin{array}{l}8.70 \mathrm{E}-12 \\
(0.000)\end{array}$ & $\begin{array}{l}2.99 \mathrm{E}-11 \\
(0.000)\end{array}$ & $\begin{array}{l}1.02 E-11 \\
(0.000)\end{array}$ \\
\hline \multicolumn{15}{|l|}{ Dermal } \\
\hline Showering & $\begin{array}{l}7.79 \mathrm{E}-04 \\
(0.523)\end{array}$ & $\begin{array}{l}8.31 \mathrm{E}-04 \\
(0.558)\end{array}$ & $\begin{array}{l}1.39 \mathrm{E}-03 \\
(0.933)\end{array}$ & $\begin{array}{l}\text { 1.80E-04 } \\
(0.121)\end{array}$ & $\begin{array}{l}1.72 \mathrm{E}-04 \\
(0.115)\end{array}$ & $\begin{array}{l}2.81 \mathrm{E}-02 \\
(18.86)\end{array}$ & $\begin{array}{l}2.07 E-05 \\
(0.014)\end{array}$ & $\begin{array}{l}9.36 \mathrm{E}-04 \\
(0.628)\end{array}$ & $\begin{array}{l}5.27 \mathrm{E}-05 \\
(0.035)\end{array}$ & $\begin{array}{l}1.19 \mathrm{E}-05 \\
(0.008)\end{array}$ & $\begin{array}{l}5.40 \mathrm{E}-05 \\
(0.036)\end{array}$ & $\begin{array}{l}2.41 \mathrm{E}-04 \\
(0.162)\end{array}$ & $\begin{array}{l}8.73 E-05 \\
(0.059)\end{array}$ & $\begin{array}{l}1.44 \mathrm{E}-06 \\
(0.001)\end{array}$ \\
\hline Soil contact & $\begin{array}{l}\text { 7.10E-08 } \\
(0.000)\end{array}$ & $\begin{array}{l}\text { 6.19E-09 } \\
(0.000)\end{array}$ & $\begin{array}{l}3.94 \mathrm{E}-08 \\
(0.000)\end{array}$ & $\begin{array}{l}2.03 E-20 \\
(0.000)\end{array}$ & $\begin{array}{l}7.98 \mathrm{E}-09 \\
(0.000)\end{array}$ & $\begin{array}{l}1.59 \mathrm{E}-07 \\
(0.000)\end{array}$ & $\begin{array}{l}\text { 6.70E-10 } \\
(0.000)\end{array}$ & $\begin{array}{l}1.44 \mathrm{E}-08 \\
(0.000)\end{array}$ & $\begin{array}{l}3.31 \mathrm{E}-10 \\
(0.000)\end{array}$ & $\begin{array}{l}1.01 \mathrm{E}-11 \\
(0.000)\end{array}$ & $\begin{array}{l}4.76 \mathrm{E}-09 \\
(0.000)\end{array}$ & $\begin{array}{l}8.67 \mathrm{E}-11 \\
(0.000)\end{array}$ & $\begin{array}{l}2.98 \mathrm{E}-10 \\
(0.000)\end{array}$ & $\begin{array}{l}3.55 \mathrm{E}-11 \\
(0.000)\end{array}$ \\
\hline \multicolumn{15}{|l|}{ Inhalation } \\
\hline Indoor & $\begin{array}{l}4.26 \mathrm{E}-03 \\
(2.859)\end{array}$ & $\begin{array}{l}2.51 \mathrm{E}-04 \\
(0.168)\end{array}$ & $\begin{array}{l}4.74 \mathrm{E}-03 \\
(3.181)\end{array}$ & $\begin{array}{l}4.26 \mathrm{E}-15 \\
(0.000)\end{array}$ & $\begin{array}{l}2.13 \mathrm{E}-03 \\
(1.430)\end{array}$ & $\begin{array}{l}2.99 \mathrm{E}-02 \\
(20.067)\end{array}$ & $\begin{array}{l}4.26 \mathrm{E}-04 \\
(0.286)\end{array}$ & $\begin{array}{l}2.49 \mathrm{E}-03 \\
(1.671)\end{array}$ & $\begin{array}{l}3.74 \mathrm{E}-04 \\
(0.251)\end{array}$ & $\begin{array}{l}2.13 \mathrm{E}-05 \\
(0.014)\end{array}$ & $\begin{array}{l}7.11 \mathrm{E}-04 \\
(0.477)\end{array}$ & $\begin{array}{l}\text { 1.49E-04 } \\
(0.100)\end{array}$ & $\begin{array}{l}1.49 \mathrm{E}-04 \\
(0.100)\end{array}$ & $\begin{array}{l}4.97 \mathrm{E}-05 \\
(0.033)\end{array}$ \\
\hline Showering & $\begin{array}{l}\text { 5.09E-04 } \\
(0.342)\end{array}$ & $\begin{array}{l}2.99 \mathrm{E}-05 \\
(0.020)\end{array}$ & $\begin{array}{l}5.65 E-04 \\
(0.379)\end{array}$ & $\begin{array}{l}5.09 \mathrm{E}-16 \\
(0.000)\end{array}$ & $\begin{array}{l}2.54 \mathrm{E}-04 \\
(0.170)\end{array}$ & $\begin{array}{l}3.56 \mathrm{E}-03 \\
(2.389)\end{array}$ & $\begin{array}{l}\text { 5.09E-05 } \\
(0.034)\end{array}$ & $\begin{array}{l}2.97 E-04 \\
(0.199)\end{array}$ & $\begin{array}{l}4.46 \mathrm{E}-05 \\
(0.030)\end{array}$ & $\begin{array}{l}2.54 \mathrm{E}-06 \\
(0.002)\end{array}$ & $\begin{array}{l}8.48 \mathrm{E}-05 \\
(0.057)\end{array}$ & $\begin{array}{l}1.78 \mathrm{E}-05 \\
(0.012)\end{array}$ & $\begin{array}{l}1.78 \mathrm{E}-05 \\
(0.012)\end{array}$ & $\begin{array}{l}5.93 \mathrm{E}--06 \\
(0.004)\end{array}$ \\
\hline Total risk & $\begin{array}{l}7.11 \mathrm{E}-03 \\
(4.772)\end{array}$ & $\begin{array}{l}3.46 \mathrm{E}-03 \\
(2.322)\end{array}$ & $\begin{array}{l}8.56 \mathrm{E}-03 \\
(5.745)\end{array}$ & $\begin{array}{l}1.72 \mathrm{E}-03 \\
(1.154)\end{array}$ & $\begin{array}{l}3.32 \mathrm{E}-03 \\
(2.228)\end{array}$ & $\begin{array}{l}8.39 \mathrm{E}-01 \\
(563.06)\end{array}$ & $\begin{array}{l}6.50 E-04 \\
(0.436)\end{array}$ & $\begin{array}{l}4.63 E-03 \\
(3.107)\end{array}$ & $\begin{array}{l}\text { 5.47E-04 } \\
(0.367)\end{array}$ & $\begin{array}{l}4.33 \mathrm{E}-05 \\
(0.029)\end{array}$ & $\begin{array}{l}1.02 \mathrm{E}-03 \\
(0.685)\end{array}$ & $\begin{array}{l}5.59 E-04 \\
(0.375)\end{array}$ & $\begin{array}{l}3.30 \mathrm{E}-04 \\
(0.221)\end{array}$ & $\begin{array}{l}1.08 \mathrm{E}-04 \\
(0.072)\end{array}$ \\
\hline
\end{tabular}

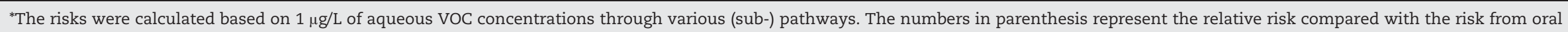
ingestion of PCE (taking the specific risk of oral water intake of PCE as unity). 
Table 5 - Specific cancer risk ${ }^{*}$ for VOCs through different pathways

\begin{tabular}{|c|c|c|c|c|c|c|}
\hline Pathway & PCE & TCE & 1,1-DCE & VC & 1,1-DCEA & BENZ \\
\hline \multicolumn{7}{|l|}{ Ingestion } \\
\hline Water & $\begin{array}{l}7.76 \mathrm{E}-07 \\
(1.00)\end{array}$ & $\begin{array}{l}1.64 \mathrm{E}-07 \\
(0.211)\end{array}$ & $\begin{array}{l}8.96 \mathrm{E}-06 \\
(11.546)\end{array}$ & $\begin{array}{l}4.03 \mathrm{E}-06 \\
(5.193)\end{array}$ & $\begin{array}{l}8.51 \mathrm{E}-08 \\
(0.110)\end{array}$ & $\begin{array}{l}1.49 \mathrm{E}-06 \\
(1.920)\end{array}$ \\
\hline Shower & $\begin{array}{l}7.76 \mathrm{E}-09 \\
(0.010)\end{array}$ & $\begin{array}{l}1.64 \mathrm{E}-09 \\
(0.002)\end{array}$ & $\begin{array}{l}8.96 \mathrm{E}-08 \\
(0.115)\end{array}$ & $\begin{array}{l}4.03 \mathrm{E}-08 \\
(0.052)\end{array}$ & $\begin{array}{l}8.51 \mathrm{E}-10 \\
(0.001)\end{array}$ & $\begin{array}{l}\text { 1.49E-08 } \\
(0.019)\end{array}$ \\
\hline Meat & $\begin{array}{l}3.14 \mathrm{E}-12 \\
(0.000)\end{array}$ & $\begin{array}{l}6.47 \mathrm{E}-13 \\
(0.000)\end{array}$ & $\begin{array}{l}4.42 \mathrm{E}-12 \\
(0.000)\end{array}$ & $\begin{array}{l}1.98 \mathrm{E}-12 \\
(0.000)\end{array}$ & $\begin{array}{l}1.79 \mathrm{E}-14 \\
(0.000)\end{array}$ & $\begin{array}{l}7.96 \mathrm{E}-13 \\
(0.000)\end{array}$ \\
\hline Milk & $\begin{array}{l}2.54 \mathrm{E}-12 \\
(0.000)\end{array}$ & $\begin{array}{l}5.08 \mathrm{E}-13 \\
(0.000)\end{array}$ & $\begin{array}{l}9.95 \mathrm{E}-12 \\
(0.000)\end{array}$ & $\begin{array}{l}4.45 \mathrm{E}-12 \\
(0.000)\end{array}$ & $\begin{array}{l}4.03 \mathrm{E}-14 \\
(0.000)\end{array}$ & $\begin{array}{l}\text { 1.79E-12 } \\
(0.000)\end{array}$ \\
\hline Vegetables & $\begin{array}{l}1.48 \mathrm{E}-08 \\
(0.019)\end{array}$ & $\begin{array}{l}4.39 \mathrm{E}-09 \\
(0.006)\end{array}$ & $\begin{array}{l}\text { 1.59E-07 } \\
(0.205)\end{array}$ & $\begin{array}{l}6.88 \mathrm{E}-08 \\
(0.089)\end{array}$ & $\begin{array}{l}7.68 \mathrm{E}-10 \\
(0.001)\end{array}$ & $\begin{array}{l}\text { 2.07E-08 } \\
(0.027)\end{array}$ \\
\hline Crops & $\begin{array}{l}1.32 \mathrm{E}-08 \\
(0.017)\end{array}$ & $\begin{array}{l}1.09 \mathrm{E}-08 \\
(0.014)\end{array}$ & $\begin{array}{l}9.00 \mathrm{E}-07 \\
(1.160)\end{array}$ & $\begin{array}{l}6.02 \mathrm{E}-08 \\
(0.078)\end{array}$ & $\begin{array}{l}8.19 \mathrm{E}-11 \\
(0.000)\end{array}$ & $\begin{array}{l}8.18 \mathrm{E}-09 \\
(0.011)\end{array}$ \\
\hline Soil & $\begin{array}{l}3.71 \mathrm{E}-12 \\
(0.000)\end{array}$ & $\begin{array}{l}1.16 \mathrm{E}-12 \\
(0.000)\end{array}$ & $\begin{array}{l}2.14 \mathrm{E}-11 \\
(0.000)\end{array}$ & $\begin{array}{l}9.61 \mathrm{E}-12 \\
(0.000)\end{array}$ & $\begin{array}{l}3.83 E-14 \\
(0.000)\end{array}$ & $\begin{array}{l}2.47 \mathrm{E}-12 \\
(0.000)\end{array}$ \\
\hline \multicolumn{7}{|l|}{ Dermal } \\
\hline Showering & $\begin{array}{l}4.05 E-07 \\
(0.522)\end{array}$ & $\begin{array}{l}6.40 \mathrm{E}-08 \\
(0.082)\end{array}$ & $\begin{array}{l}7.48 \mathrm{E}-06 \\
(9.639)\end{array}$ & $\begin{array}{l}1.52 \mathrm{E}-07 \\
(0.196)\end{array}$ & $\begin{array}{l}1.18 \mathrm{E}-08 \\
(0.015)\end{array}$ & $\begin{array}{l}1.60 \mathrm{E}-06 \\
(2.062)\end{array}$ \\
\hline Soil contact & $\begin{array}{l}3.69 \mathrm{E}-11 \\
(0.000)\end{array}$ & $\begin{array}{l}1.16 \mathrm{E}-11 \\
(0.000)\end{array}$ & $\begin{array}{l}2.13 \mathrm{E}-10 \\
(0.000)\end{array}$ & $\begin{array}{l}8.60 \mathrm{E}-13 \\
(0.000)\end{array}$ & $\begin{array}{l}3.82 \mathrm{E}-13 \\
(0.000)\end{array}$ & $\begin{array}{l}2.46 \mathrm{E}-11 \\
(0.000)\end{array}$ \\
\hline \multicolumn{7}{|l|}{ Inhalation } \\
\hline Indoor & $\begin{array}{l}8.53 E-08 \\
(0.110)\end{array}$ & $\begin{array}{l}2.56 \mathrm{E}-07 \\
(0.330)\end{array}$ & $\begin{array}{l}7.68 \mathrm{E}-06 \\
(9.897)\end{array}$ & $\begin{array}{l}1.15 \mathrm{E}-05 \\
(14.820)\end{array}$ & $\begin{array}{l}2.43 E-07 \\
(0.313)\end{array}$ & $\begin{array}{l}4.26 \mathrm{E}-06 \\
(5.490)\end{array}$ \\
\hline Showering & $\begin{array}{l}1.02 \mathrm{E}-08 \\
(0.013)\end{array}$ & $\begin{array}{l}3.05 E-08 \\
(0.039)\end{array}$ & $\begin{array}{l}9.16 \mathrm{E}-07 \\
(1.180)\end{array}$ & $\begin{array}{l}1.37 \mathrm{E}-06 \\
(1.765)\end{array}$ & $\begin{array}{l}2.90 \mathrm{E}-08 \\
(0.037)\end{array}$ & $\begin{array}{l}5.09 \mathrm{E}-07 \\
(0.656)\end{array}$ \\
\hline Total risk & $\begin{array}{l}1.31 \mathrm{E}-06 \\
(1.688)\end{array}$ & $\begin{array}{l}5.31 \mathrm{E}-07 \\
(0.684)\end{array}$ & $\begin{array}{l}2.62 \mathrm{E}-05 \\
(33.763)\end{array}$ & $\begin{array}{l}1.72 \mathrm{E}-05 \\
(22.192)\end{array}$ & $\begin{array}{l}3.71 \mathrm{E}-07 \\
(0.478)\end{array}$ & $\begin{array}{l}\text { 7.91E-06 } \\
(10.193)\end{array}$ \\
\hline
\end{tabular}

*The risks were calculated based on $1 \mu \mathrm{g} / \mathrm{L}$ of aqueous VOC concentrations through various (sub-) pathways. The number in the parenthesis represents the relative risk calculated by comparing the risk to that from oral ingestion of PCE.

For 1,1-DCE, the total specific cancer risk was $2.62 \times 10^{-5}$, most of which $\left(8.96 \times 10^{-6}\right)$ came from exposure through water ingestion. Exposure to 1,1-DCE through indoor breathing and dermal absorption when showering also resulted in observa- ble specific cancer risks. In contrast, exposure to this chemical through ingestion of meat, milk, and soil, and dermal absorption by soil contact, all made negligible contributions to the specific cancer risk.

\begin{tabular}{|c|c|c|c|c|c|c|c|c|c|c|c|}
\hline \multirow{2}{*}{$\begin{array}{l}\text { Sampling } \\
\text { location }\end{array}$} & \multirow{2}{*}{$\begin{array}{l}\text { Sampling } \\
\text { time }\end{array}$} & \multicolumn{9}{|c|}{ VOCs } & \multirow{2}{*}{$\begin{array}{c}\text { Total } \\
\text { risk }\end{array}$} \\
\hline & & PCE & TCE & 1,1-DCE & cis-1,2-DCE & VC & 1,1-DCEA & BENZ & TLN & 1,2-DCB & \\
\hline S6 & April & - & $\begin{array}{l}5.20 \mathrm{E}-7 \\
(3.39 \mathrm{E}-3)\end{array}$ & - & - & - & - & - & - & - & $\begin{array}{l}5.20 \mathrm{E}-7 \\
(3.39 \mathrm{E}-3)\end{array}$ \\
\hline S2 & April & - & - & - & - & - & - & - & $\begin{array}{l}- \\
5.47 \mathrm{E}-4\end{array}$ & - & - \\
\hline \multirow[t]{2}{*}{ S8 } & April & $\begin{array}{l}2.62 \mathrm{E}-6 \\
(1.42 \mathrm{E}-2)\end{array}$ & $\begin{array}{l}1.06 \mathrm{E}-6 \\
(6.92 \mathrm{E}-3)\end{array}$ & $\begin{array}{l}3.93 \mathrm{E}-4 \\
(1.28 \mathrm{E}-1)\end{array}$ & $-\overline{1.72 \mathrm{E}-3}$ & - & $\begin{array}{l}3.71 \mathrm{E}-7 \\
(6.50 \mathrm{E}-4)\end{array}$ & - & - & - & $\begin{array}{l}3.97 \mathrm{E}-4 \\
(1.51 \mathrm{E}-1)\end{array}$ \\
\hline & Jul. & $\begin{array}{l}5.24 \mathrm{E}-6 \\
(2.84 \mathrm{E}-2)\end{array}$ & $\begin{array}{l}1.59 \mathrm{E}-6 \\
(1.04 \mathrm{E}-2)\end{array}$ & $\begin{array}{l}6.29 E-4 \\
(2.05 E-1)\end{array}$ & $-\overline{3.44 \mathrm{E}-3}$ & - & $\begin{array}{l}7.42 \mathrm{E}-7 \\
(1.30 \mathrm{E}-3)\end{array}$ & - & - & - & $\begin{array}{l}6.37 \mathrm{E}-4 \\
(2.49 \mathrm{E}-1)\end{array}$ \\
\hline \multirow[t]{3}{*}{ S7 } & April & $\begin{array}{l}2.75 \mathrm{E}-5 \\
(1.49 \mathrm{E}-1)\end{array}$ & $\begin{array}{l}1.23 \mathrm{E}-4 \\
(7.99 \mathrm{E}-1)\end{array}$ & $\begin{array}{l}4.98 \mathrm{E}-4 \\
(1.63 \mathrm{E}-1)\end{array}$ & - & $\begin{array}{l}1.11 \mathrm{E}-2 \\
(5.46 \mathrm{E}+2)\end{array}$ & $\begin{array}{l}9.65 \mathrm{E}-6 \\
(1.69 \mathrm{E}-2)\end{array}$ & $\begin{array}{l}2.37 \mathrm{E}-5 \\
(1.39 \mathrm{E}-2)\end{array}$ & - & $\begin{array}{l}- \\
(7.14 \mathrm{E}-3)\end{array}$ & $\begin{array}{l}2.18 E-2 \\
(5.47 E+2)\end{array}$ \\
\hline & July & $\begin{array}{l}4.11 \mathrm{E}-6 \\
(2.23 \mathrm{E}-2)\end{array}$ & $\begin{array}{l}6.64 \mathrm{E}-6 \\
(4.33 \mathrm{E}-2)\end{array}$ & $\begin{array}{l}5.61 \mathrm{E}-4 \\
(1.83 \mathrm{E}-1)\end{array}$ & - & $\begin{array}{l}7.31 E-5 \\
(3.57 E+0)\end{array}$ & $\begin{array}{l}2.85 \mathrm{E}-6 \\
(5.00 \mathrm{E}-3)\end{array}$ & - & - & - & $\begin{array}{l}6.48 \mathrm{E}-4 \\
(3.82 \mathrm{E}+0)\end{array}$ \\
\hline & Oct. & $\begin{array}{l}5.40 \mathrm{E}-6 \\
(2.93 \mathrm{E}-2)\end{array}$ & $\begin{array}{l}1.64 \mathrm{E}-5 \\
(1.07 \mathrm{E}-1)\end{array}$ & $\begin{array}{l}3.41 \mathrm{E}-4 \\
(1.11 \mathrm{E}-1)\end{array}$ & $\begin{array}{l}- \\
1.42 \mathrm{E}-1\end{array}$ & $\begin{array}{l}8.18 \mathrm{E}-3 \\
(3.99 \mathrm{E}+2)\end{array}$ & $\begin{array}{l}2.09 \mathrm{E}-5 \\
(3.65 \mathrm{E}-2)\end{array}$ & $\begin{array}{l}1.67 \mathrm{E}-4 \\
(9.77 \mathrm{E}-2)\end{array}$ & - & - & $\begin{array}{l}8.73 E-3 \\
(4.00 E+2)\end{array}$ \\
\hline \multirow[t]{2}{*}{ W2 } & April & - & - & - & - & - & - & - & $-\overline{8.91 \mathrm{E}-4}$ & - & - \\
\hline & Oct. & - & - & - & - & - & - & - & $\begin{array}{l}- \\
1.10 \mathrm{E}-2\end{array}$ & - & - \\
\hline $\begin{array}{l}\text { Risk based } \\
\text { DW standar }\end{array}$ & & $\begin{array}{l}6.55 \mathrm{E}-6 \\
(3.56 \mathrm{E}-2)\end{array}$ & $\begin{array}{l}2.66 \mathrm{E}-6 \\
(1.73 \mathrm{E}-3)\end{array}$ & $\begin{array}{l}1.83 \mathrm{E}-4 \\
(5.99 \mathrm{E}-2)\end{array}$ & $-\overline{1.20 \mathrm{E}-1}$ & $\begin{array}{l}3.44 \mathrm{E}-5 \\
(1.68 \mathrm{E}+0)\end{array}$ & $\begin{array}{l}1.86 \mathrm{E}-5 \\
(3.25 \mathrm{E}-2)\end{array}$ & $\begin{array}{l}3.96 \mathrm{E}-5 \\
(2.32 \mathrm{E}-2)\end{array}$ & $-\overline{5.47 \mathrm{E}-1}$ & $\begin{array}{l}- \\
6.12 \mathrm{E}-1\end{array}$ & $\begin{array}{l}3.18 E-4 \\
(9.31 E+0)\end{array}$ \\
\hline
\end{tabular}

The numbers in the table represent the cancer risks, and the numbers in parentheses represent the non-cancer risks. 
For VC, indoor breathing was the most important pathway for cancer risk; water ingestion and dermal absorption when showering also made appreciable contributions to cancer risk associated with this chemical.

For BENZ, indoor inhalation and dermal absorption when showering were the two major pathways for cancer risk.

Comparisons among the various exposure pathways revealed that indoor breathing, water ingestion, and dermal absorption when showering were the three most important pathways for cancer risk development. Of the various exposure pathways that involved oral ingestion, water intake resulted in the highest cancer risk, with ingestion of vegetables and crops ranking second and third respectively. However, in general terms, the findings with respect to cancer risk through the food chain were similar to those for noncancer risk through the food chain-that is, among the investigated carcinogenic VOCs, cancer risk contribution through the food chain was minimal (apart from the risk associated with direct water ingestion). Dermal absorption through soil contact and ingestion of meat, milk, and soil were found to have negligible influences on specific cancer risk.

\subsubsection{Selection of VC skin permeability coefficient}

As previously noted, the selection of parameters is of crucial importance in any calculation of risk. In the case of dermal absorption pathways, the skin permeability coefficient $\left(k_{\mathrm{p}}\right)$ is the key factor in determining the amount of a chemical that penetrates the skin. In accordance with USEPA (1992), the present study used a $k_{\mathrm{p}}$ of $7.3 \times 10^{-3}$ to calculate the risks asso- ciated with dermal absorption of VC. These are the results that have been presented in Tables 4 and 5. In contrast, CDTSC (2008) has suggested that a $k_{\mathrm{p}}$ factor of $8.12 \times 10^{-1}$ should be utilised, which is two orders of magnitude greater than that used in this study.

Using the $k_{\mathrm{p}}$ suggested by USEPA (1992), the non-cancer risk from dermal absorption of showering water was $2.81 \times 10^{-2}$ and that from soil was $1.59 \times 10^{-7}$; however, utilising the $k_{\mathrm{p}}$ suggested by CDTSC (2008), these non-cancer risks were $3.13 \times 10^{-5}$ and $1.77 \times 10^{-5}$ respectively. Similarly, using the $k_{\mathrm{p}}$ proposed by CDTSC (2008) the calculated cancer risks from dermal absorption were $1.69 \times 10^{-5}$ for showering water and $9.57 \times 10^{-11}$ for soil, which were approximately two orders of magnitude greater than those using the $k_{\mathrm{p}}$ suggested by USEPA (which were $1.52 \times 10^{-7}$ for showering water and $8.60 \times 10^{-13}$ for soil).

When the $k_{\mathrm{p}}$ suggested by CDTSC (2008) is used for risk calculation, VC risk from dermal absorption of showering water becomes the most significant pathway. This finding is quite unusual because, in most cases, inhalation exposure is considered the most dominant pathway for VOCs. Moreover, the known chemical characteristics of VC (such as its molecular weight and its chemical partition characteristics) do not support the suggestion that dermal absorption of VC through showering water might be the most dominant pathway. For these reasons, the $k_{\mathrm{p}}$ proposed by USEPA (1992) was considered a more appropriate skin permeability coefficient than that proposed by CDTSC (2008) for the calculation of VC risk in the present study.

Table 7 - Calculated concentrations of VOCs ${ }^{\text {a }}$ based on general guidance values and groundwater criteria of VOCs in many countries/organization around the world

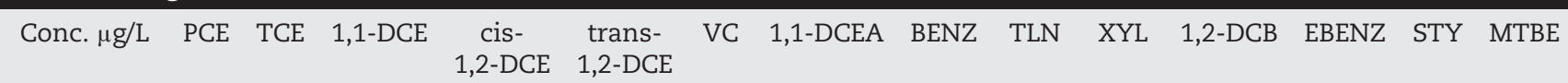

\begin{tabular}{|c|c|c|c|c|c|c|c|c|c|c|c|c|c|c|}
\hline \multicolumn{15}{|c|}{ All exposure pathways considered } \\
\hline For cancer & 0.76 & 1.88 & 0.04 & & & 0.06 & 2.70 & 0.13 & & & & & & \\
\hline $\begin{array}{l}\text { For } \\
\text { non-cancer }\end{array}$ & 141 & 289 & 117 & 581 & 301 & 1 & 1539 & 216 & 1828 & 23091 & 981 & 1790 & 3030 & 9285 \\
\hline \multicolumn{15}{|c|}{ Selected exposure pathways considered } \\
\hline For cancer & 0.79 & 2.07 & 0.04 & & & 0.06 & 2.94 & 0.14 & & & & & & \\
\hline $\begin{array}{l}\text { For } \\
\text { non-cancer }\end{array}$ & 153 & 311 & 128 & 599 & 328 & 1 & 1679 & 233 & 1995 & 24594 & 1074 & 1855 & 3216 & 9907 \\
\hline \multicolumn{15}{|l|}{ Taiwan $^{\mathrm{b}}$} \\
\hline Type I & 5 & 5 & 7 & 70 & 100 & 2 & & 5 & 1000 & & & & & \\
\hline Type II & 50 & 50 & 70 & 700 & 1000 & 20 & & 50 & 10000 & & & & & \\
\hline US EPA & 5 & 5 & 7 & 70 & 1000 & 2 & 5 & 5 & 1000 & 10000 & 600 & 700 & 100 & \\
\hline US NJ & 1 & 1 & 2 & 70 & 100 & 2 & 2 & 1 & & 1000 & 600 & 700 & 100 & 70 \\
\hline US California & & & & & & & 3 & & & & & & & \\
\hline US Florida & 3 & 3 & 7 & 70 & 100 & 1 & & 1 & 1000 & 10000 & 600 & 700 & 100 & 50 \\
\hline WHO & 40 & 70 & 30 & 50 & 100 & 3 & & 10 & 700 & 500 & 1000 & 300 & 20 & \\
\hline EU & & & & & & 0.5 & & 1 & & & & & & \\
\hline England & & & & & & 0.5 & & & & & & & & \\
\hline Japan & 10 & 30 & 20 & 40 & 100 & & & 1 & & 400 & & & & \\
\hline Australia & 50 & & & & & 0.3 & & 1 & 800 & 600 & 1500 & 300 & 30 & \\
\hline Canada & & 50 & & & & 2 & & 5 & & 300 & 200 & 2.4 & & \\
\hline
\end{tabular}

a The suggested VOCs groundwater criteria were calculated to meet the general guidance values (both for non-cancer and cancer risks) based on the accumulative specific risks from all and selected exposure pathways. The selected exposure pathways include water ingestion, dermal absorption when showering, and indoor inhalation.

b Type I indicates the groundwater within the source water protection zones in Taiwan, and Type II indicates those water bodies other than the Type I. 


\subsection{Total health risks from exposure to groundwater}

Using the VOC concentrations shown in Table 3, the total noncancer and cancer risks from exposure to VOCs in groundwater were calculated. The results are presented in Table 6.

The calculations revealed that the three groundwater samples from S7 contained the most VOCs and posed the most significant threats to the public. The overall non-cancer risks for these samples were $4.47 \times 10^{2}$ (April 2005), $3.82 \times 10^{2}$ (July 2005), and $4.00 \times 10^{2}$ (October 2005), and the overall cancer risks were $2.18 \times 10^{-2}$ (April 2005), $6.48 \times 10^{-4}$ (July 2005), and $8.73 \times 10^{-3}$ (October 2005). All of these risks exceeded the generally accepted criteria for threats to public health.

The VOCs in groundwater samples from S8 $\left(3.97 \times 10^{-4}\right.$ for April 2005 and $6.37 \times 10^{-4}$ for July 2005) also represented a demonstrable cancer risk.

In contrast, the groundwater samples collected from S2, S6, and W2 revealed only small amounts of VOCs, which did not represent a comparable threat to public health.

Table 6 also shows the non-cancer and cancer risks in terms of the VOC criteria for drinking water. For non-cancer risks, VC was the only pollutant with a risk higher than the general guidance risk value. For cancer risks, all six carcinogenic VOCs had demonstrable cancer risks higher than the general guidance risk value. In other words, groundwater can still exhibit appreciable cancer risk even if the VOCs in that groundwater meet the water-quality criteria. For example, if the concentration of 1,1-DCE is at a level of $0.007 \mathrm{mg} / \mathrm{L}$ (which is the current Taiwan drinking water criterion), it might pose a significant carcinogenic threat to the public compared with other VOCs (about 183 times higher than the general guidance cancer risk value). The risk would be even higher if more than one VOC were found in the groundwater.

\subsection{Correspondence between VOC groundwater concentrations and general guidance values}

Because the quality criteria for drinking water might not be able to ensure the effective safety of groundwater utilisation, amendment of the current VOC drinking-water criteria might be necessary to protect public health. To investigate this contention, the maximum allowable groundwater concentrations for each VOC were calculated on the basis of the general guidance values for non-cancer and cancer risk assessment taking all exposure pathways into consideration. For groundwater VOC concentrations at the calculated value, the total risk from all exposure pathways was equal to the recommended guidance value (that is, 1.00 for non-cancer risk and $10^{-6}$ for cancer risk). These calculated concentrations were then compared with the current quality criteria for drinking water. Table 7 summarises the calculated VOC drinking water criteria based on the specific cancer and non-cancer risks at their guidance values. Table 7 also shows the VOC drinking water quality criteria of several other countries and organisations.

From the perspective of non-cancer risk control for groundwater, most (but not all) of the drinking water criteria appear to be adequate to ensure the safe utilisation of groundwater; the exceptions are those for VC in the standards of the USA, WHO, and Canada, and those for 1,2-DCB in the standards of WHO and Australia. In contrast, with regard to cancer risk, if the calculated VOC concentrations for suggested cancer risk $\left(10^{-6}\right)$ are compared to the water-quality criteria, most (but not all) of the criteria are not suitable for protection of the general public; the exceptions are those for 1,1-DCEA in two of the USA standards (EPA and California) and those for TCE in the USA (NJ) standard. Because continuous consumption of groundwater that barely meets the water-quality criteria can constitute a significant cancer risk, it is apparent from these results that amendment to drinking-water quality criteria is necessary to ensure the safety of groundwater.

It is noteworthy that the present study evaluated the drinking-water criteria by a risk calculation that considered all exposure pathways, which might have led to the more stringent standards for groundwater quality management suggested here. For example, the significant risk-exposure pathways for VOC are generally considered to be indoor breathing, water ingestion, and dermal absorption. If only some of these important exposure pathways are considered, the total risk for a given VOC could be underestimated and the resulting waterquality criteria could be inadequate. In general, the accumulated risk resulting from the major exposure pathways accounts for $90 \%$ of the total risk. By considering only the three important exposure pathways, the maximum groundwater concentrations allowed for each VOC to meet the non-cancer and cancer general guidance values were calculated, and presented in Table 7. The calculated concentrations with the three most important risk-contributing exposure pathways were comparable (within 10\% difference) to those with all investigated exposure pathways. For future application, it is suggested that employment of the three most important exposure pathways (water ingestion, dermal absorption when showering, and indoor breathing) is likely to provide satisfactory assessment for non-cancer and cancer risk evaluation.

\section{Conclusions}

This study has undertaken a risk assessment of exposure to volatile organic compounds (VOCs) in groundwater in Taiwan by investigating the specific non-cancer and cancer risks of exposure to $1 \mu \mathrm{g} / \mathrm{L}$ of various VOCs through a variety of pathways.

The study has found that the highest specific non-cancer risk was associated with water ingestion of vinyl chloride (VC) $\left(7.46 \times 10^{-1}\right)$ and that the highest specific cancer risk was associated with indoor inhalation of VC $\left(1.15 \times 10^{-5}\right.$, which exceeded the general guidance value of $\left.10^{-6}\right)$. The secondhighest specific non-cancer risk was associated with exposure to VC by indoor breathing $\left(2.99 \times 10^{-2}\right)$ and the second-highest specific cancer risk was associated with 1,1-dichloroethylene (1,1-DCE) exposure through water ingestion $\left(8.96 \times 10^{-6}\right)$. In addition, demonstrable risks were apparent in association with indoor breathing of 1,1-DCE, tetrachloroethylene (PCE), and benzene (BENZ). The study found that xylene (XYL), methyl-t-butyl ether (MTBE), and styrene (STY) made the least significant contributions to non-cancer risk, and that 1,1dichloroethane (1,1-DCEA) and trichloroethylene (TCE) made the least significant contributions to cancer risk.

Among the investigated exposure pathways, the most significant risk-contributing exposure pathways for VOC were 
indoor breathing, water ingestion, and dermal absorption when showering. Risks from exposure through ingestion of meat, milk, soil, and showering water were found to be insignificant.

In general, the groundwater analysed in the present study does not pose a significant threat to the public, apart from the areas with abundant contamination sources. In particular, the groundwater around S7 (sampled in April 2005) contained appreciable amounts of VOCs with the potential to cause significant non-cancer and cancer risks $\left(547 \times 10^{-2}\right.$ and $2.18 \times 10^{-2}$, respectively). The S8 sample of July 2005 also exhibited significant cancer risk $\left(6.37 \times 10^{-4}\right)$. All of the significant risks were associated with chlorinated VOCs and benzene in groundwater.

The results of the study have demonstrated that the current quality criteria for VOCs in drinking water might not be capable of ensuring safe utilisation of groundwater in areas where groundwater is used as the primary water supply. Whereas most drinking-water criteria are established in accordance with all exposure pathways, the study suggests that inclusion of only the more important pathways in the risk assessment might provide a comparable risk calculation while simplifying the process of risk assessment. However, it should be noted that, in setting drinking-water standards, various agencies employ different models with different input parameters. Their evaluations of drinking-water maximum contaminant levels might well be sound with respect to the models they employ.

\section{Acknowledgment}

This study was supported by Taiwan EPA via Project number EPA-94-G102-02-214.

\section{R E F E R E N C E S}

Barry DA, Prommer H, Miller CT, Engesgaard P, Brun A, Zheng C. Modelling the fate of oxidizable organic contaminants in groundwater. Adv Water Resour 2002;25:945-83.

Belpomme D, Irigaray P, Hardell L, Clapp R, Montagnier L, Epstein $\mathrm{S}$, et al. The multitude and diversity of environmental carcinogens. Environ Res 2007;105:414-29.

CDTSC, website information, www.dtsc.ca.gov/AssessingRisk/ ctox_dwn.cfm, May, 2008.

Chen Y-C, Ma H-W. Model comparison for risk assessment: a case study of contaminated groundwater. Chemosphere 2006;63:751-61.

Claxton LD, Woodall Jr GM. A review of the mutagenicity and rodent carcinogenicity of ambient air. Rev Mutat Res 2007;636:36-94.

DOH. Nutrition and Health Survey in Taiwan, 1993 1996. Taipei, R.O.C.: Department of Health; 2000.

Droppo JA, Whelan G, Buck JW, Strenge DL. Supplemental Mathematical Formulations: The Multimedia Environmental Pollutant Assessment System (MEPAS). Richland, Washington, USA: Pacific Northwest Laboratory; 1989.

EALTEPA. Website information, http://www.niea.gou.tw/niea/ WATER/W10251C.htm, Environmental Analysis Laboratory, Taiwan EPA, Nov. 2008 (in Chinese).
Edil RB. A review of aqueous-phase VOC transport in modern landfill liners. Waste Manage 2003;23:561-71.

Ellis PA, Rivett MO. Assessing the impact of VOC-contaminated groundwater on surface water at the city scale. J Contam Hydrol 2007;91:107-27.

Guo H, Lee SC, Chan LY, Li WM. Risk assessment of exposure to volatile organic compounds in different indoor environment. Environ Res 2004;94:57-66.

Hohener P, Werner D, Baisiger C, Pasteris G. Worldwide occurrence and fate of chlorofluorocarbons in groundwater. Crit Rev Environ Sci Technol 2003;33:1-29.

Kistemann T, Hundhausen J, Herbst S, Calben T, Farber H. Assessment of a groundwater contamination with vinyl chloride (VC) and precursor volatile organic compounds (VOC) by use of a geographical information system (GIS). Int J Hyg Environ Health 2007. doi:10.1016/j.ijheh.2007.02.011.

Kuo H-W, Chiang T-F, Lo I-I, Lai J-S, Chan C-C, Wang J-D. Estimates of cancer risk from chloroform exposure during showering in Taiwan. Sci Total Environ 1998;218:1-7.

Liang H-M, Liao C-M. Modelling VOC-ordor exposure risk in livestock buildings. Chemosphere 2007;68:781-9.

Malherbe L, Mandin C. VOC emissions during outdoor ship painting and health-risk assessment. Atmos Environ 2007;41:6322-30.

Mckone TE. CalTOX: A multimedia total-exposure model for hazardous waste sites: Part I: executive summary. Department of Toxic Substances Control, Lawrence Livermore National Laboratory, Livermore, California, USA; 1993.

MOEA. Groundwater monitoring network plan in Taiwan. Water Resources Bureau, Ministry of Economic Affairs, Taipei, Taiwan; 1997.

Posen P, Lovett A, Hiscock K, Ever S, Ward R, Reid B. Incorporating variations in preticide catabolic activity into GIS-based groundwater risk assessment. Sci Total Environ 2006;367:641-52.

TEPA. Studies on Soil and Groundwater Contamination Remediation Act and health risk assessment principle - soil and groundwater contamination sites health risk assessment evaluation principle. EPA-93-G102-02-211 Taiwan EPA project report; 2005a. (in Chinese).

TEPA. Investigation and evaluation of volatile organic compounds in groundwater. EPA-94-G102-02-214 Taiwan EPA project report; 2005b. (in Chinese).

TEPA. Guideline for Parameter Selection, Principle of Soil and Groundwater Contamination Site Risk Assessment. Taipei, R.O.C: Environmental Protection Administration; 2005c (in Chinese).

Ton C-D, Exposure and Health Risk Assessment of Groundwater Contamination - A Case Study of Contamination Site of Tao-Yuan RCA. Master Thesis, National Taiwan University. 1999 (in Chinese).

USEPA. Risk Assessment Guidance for Superfund: Human Health Evaluation Manual, vol. 1. Washington DC, USA: Office of Emergency and Remedial Response; 1989. (Part A).

USEPA. Dermal exposure assessment: principles and applications. Office of Health and Environmental Assessment. Washington DC: USEPA; 1992.

USEPA. MMSOILS Model: Multimedia Contaminated Fate, Transport, and Exposure Model: Documentation and User's Manual Version 4.0. Washington DC, USA: Office of Research and Development; 1996.

Wang G-S, Deng Y-C, Lin T-F. Cancer risk assessment from trihalomethanes in drinking water. Sci Total Environ 2007;387:86-95. 